\title{
Secukinumab Attenuates Neuroinflammation and Neurobehavior Defect via PKC $\beta$ /ERK/NF-KB Pathway in a Rat Model of GMH
}

\section{Shengpeng Liu}

Shenzhen People's Hospital https://orcid.org/0000-0002-6618-977X

\section{Shuixiang Deng}

Huashan Hospital Fudan University

\section{Yan Ding}

Loma Linda University School of Medicine

Jerry J. Flores

Loma Linda University School of Medicine

Xiaoli Zhang

Shenzhen People's Hospital

\section{Xiaojing Jia}

Shenzhen People's Hospital

Xiao Hu

Loma Linda University School of Medicine

Jun Peng

Shenzhen People's Hospital

\section{Gang Zuo}

Loma Linda University School of Medicine

John H. Zhang

Loma Linda University School of Medicine

\section{Ye Gong}

Fudan University Huashan Hospital Department of Neurosurgery

Jiping Tang ( $\nabla_{\text {jtang@llu.edu })}$

Loma Linda University School of Medicine https://orcid.org/0000-0002-2392-9280

\section{Research}

Keywords: Secukinumab, neurobehavior, neuroinflammation, IL-17A

Posted Date: October 11th, 2021

DOl: https://doi.org/10.21203/rs.3.rs-957577/v1 
License: (c) (i) This work is licensed under a Creative Commons Attribution 4.0 International License. Read Full License 


\section{Abstract}

Aims

Germinal matrix hemorrhage $(\mathrm{GMH})$ is a disastrous clinical event for newborns. Neuroinflammation plays an important role in the development of neurological deficits after GMH. The purpose of this study is to investigate the anti-inflammatory role of secukinumab after $\mathrm{GMH}$ and its underlying mechanisms involving $\mathrm{PKC} \beta / \mathrm{ERK} / \mathrm{NF}-\mathrm{KB}$ signaling pathway.

\section{Methods}

A total of 154 Sprague-Dawley P7 rat pups were used. GMH was induced by intraparenchymal injection of bacterial collagenase. Secukinumab was administered intranasally post-GMH. PKC $\beta$ activator PMA and p-ERK activator Ceramide C6 were administered intracerebroventricularly at 24h prior to GMH induction, respectively. Neurobehavioral tests, Western blot and immunohistochemistry were used to evaluate the efficacy of secukinumab in both short-term and long-term studies.

Results

Endogenous IL-17A, IL-17RA, PKC $\beta$ and p-ERK were increased after GMH. Secukinumab treatment improved short- and long-term neurological outcomes, reduced the expression of MPO and Iba- 1 in the perihematoma area, and inhibited the expression of proinflammatory factors, such as NF-KB, IL-1 $3, T N F-a$ and IL-6. Additionally, PMA and ceramide C6 abolished the beneficial effects of secukinumab.

\section{Conclusion}

Secukinumab treatment suppressed neuroinflammation and attenuated neurological deficits after $\mathrm{GMH}$, which was mediated through the downregulation of the PKC $/$ ERK/NF-KB pathway. Secukinumab treatment may provide a promising therapeutic strategy for $\mathrm{GMH}$ patients.

\section{Introduction}

Germinal matrix hemorrhage $(\mathrm{GMH})$ is a devastating clinical event for neonates, especially for preterm or low-birthweight infants [1, 2]. Its peculiarity of high morbidity and mortality brought heavy burden to the society and survivor's family[3]. It could be predictable that the morbidity would increase as a result of the delivery of higher numbers of premature newborns, due to the improvements of diagnostic tools, reproductive technology and intensive care strategies[4]. Blood components from the ruptured immature vessels are the primary cause of acute and secondary brain injury[1]. Hence, survivors suffer from debilitating consequences, such as neurological deficits, post-hemorrhagic hydrocephalus and developmental delay $[5,6]$. However, there is no effective management for this severe disease other than prophylactic medications and surgical shunts with significant side effects[7]. Therefore, more studies are urgently needed to investigate innovative therapeutic modalities. 
The activation of inflammation cascades plays a critical role during the pathological process and aggravates neurobehavioral deficits after $\mathrm{GMH}$ [8-10]. Meanwhile, some inflammatory markers can be seen elevated in the severely hemorrhagic brain[11]. Inhibition of neuroinflammation is expected to be an effective method to reduce brain injury induced by GMH. Microglia, the main local immune cells, produces inflammatory cytokines and chemokines to elicit neuroinflammation responses [12,13]. Mounting studies uncovered that microglia function as an important inflammatory mediator between secondary brain injuries after hemorrhage [14-16].

IL-17 family has 6 members (IL-17A to IL-17F), among which IL-17A is the predominant one with wellstudied function $[17,18]$. Some evidence has shown that IL-17A is closely associated with neuroinflammation [19-21]. Also, it has been reported that serum IL-17A would increase in hemorrhagic patients and experimental animals $[19,22]$. Previous studies indicated that the protein kinase $C$ beta (PKC $\beta$ ) could be up-regulated by IL-17A and its receptor (IL-17RA)[23]. Meanwhile, PKCB/ERK pathway has been confirmed in many pathologic processes [24-26]. It has been reported that activated ERK is closely related with NF-kB[27, 28], which plays a pivotal role in inflammation cascades[29-31]. Secukinumab, a recombinant monoclonal antibody[32], specifically inhibits IL-17A. It is currently used as a treatment for some chronic immune diseases, such as psoriasis and arthritis [33, 34].

Thus, based on the evidence above, we hypothesized that the inhibition of IL-17A, via secukinumab, would potentially reduce neuroinflammation through IL-17RA/PKC $/$ ERK/NF-KB pathway in microglia, thereby improving short-term and long-term neurological outcomes after $\mathrm{GMH}$ in rats.

\section{Materials And Methods}

\subsection{Animals and GMH model}

All procedures were approved by the Institutional Animal Care and Use Committee at Loma Linda University, and in accordance with United States Public Health Service Policy on Human Care and Use of Laboratory Animals.

A total of one hundred and fifty Sprague Dawley (SD) rat pups were purchased from Envigo (Livermore, CA, USA). After arrival, rat pups were housed with their dam and littermates until sacrifice or weaning. GMH surgeries were performed on P7 pups since their brains are comparable to 30-32 weeks of human gestational age. After weaning, they were separated into different cages according to the gender. All animals were housed with controlled appropriate humidity and temperature and a $12 \mathrm{~h}$ light/dark cycle. All animals had free access to water and food.

GMH model was conducted by collagenase as described previously[35]. Briefly, GMH pups were fixed on a stereotaxic platform after being anesthetized with $2 \%-3 \%$ isoflurane (mixed with oxygen gas and air). A $10 \mu$ l syringe (Hamilton Co., Reno, NV, USA) was used to inject 0.3 units of collagenase VII-S (Sigma, ST Louis, MO, USA) at the point of $1.6 \mathrm{~mm}$ right, $1.5 \mathrm{~mm}$ rostral and $2.7 \mathrm{~mm}$ deep from the bregma guided by a microinfusing pump (Hamilton Co., Reno, NV, USA). After the injection, the needle remained in place for 
5 minutes and was withdrawn over additional 5 minutes to prevent leakage. Then the burr hole was sealed with bone wax and skin was sutured. The respiratory rate, heart-beat, color of skin, myodynamia and body temperature were monitored before recovery from anesthesia. All the pups were placed back to their mother and littermates until sacrifice. Sham pups underwent the same surgical procedures without collagenase infusion.

\subsection{Experimental design and groups}

Both male and female rat pups were randomly divided into the following experimental groups: sham, $\mathrm{GMH}, \mathrm{GMH}+$ vehicle, $\mathrm{GMH}$ + secukinumab, $\mathrm{GMH}$ + secukinumab + PMA, GMH + secukinumab + Ceramide C6, and GMH + secukinumab + DMSO. Figure 1 demonstrated the experimental design.

Experiment 1. To detect the time course of IL-17A, IL-17RA, PKCßand p-ERK, and to confirm the cellular localization of IL-17RA after GMH. Western blot (WB) was used to analyze the expression of these aforementioned proteins in the whole brain at $6 \mathrm{~h}, 12 \mathrm{~h}, 24 \mathrm{~h}, 3 \mathrm{~d}$ and $7 \mathrm{~d}$ after $\mathrm{GMH}$. Immunofluorescence was used to confirm IL-17RA expression on microglia at 24h after GMH.

Experiment 2. To investigate the effects of secukinumab on short-term and long-term neurobehavior. Negative geotaxis and righting reflex were employed to test short-term neurobehavior deficits, while Morris water maze, rotarod, and foot fault were employed to assess long-term neurobehavior deficits.

Experiment 3. To observe the effects of secukinumab on expression of inflammatory markers. Immunofluorescence was used to detect IL-1 $\beta$, MPO and Iba- 1 at $24 \mathrm{~h}$ after GMH.

Experiment 4. To investigate the mechanism of secukinumab treatment involving IL-17RA/PKC $\beta / E R K / N F-$ $K B$ signaling pathway, PKC $\beta$ activator PMA and $p$-ERK activator Ceramide $C 6$ were used. Expression levels of IL-17RA, PKC $\beta$, p-ERK, ERK, IL-1 $\beta, T N F-\alpha$ and IL- 6 were detected by Western blot.

\subsection{Drug administration}

Based on previous experiments[36], secukinumab (TM-Secu-00002-6; TheraMabs BioTechnology Inc., China) at the dosages of $0.6 \mathrm{mg} / \mathrm{kg}$ or phosphate-buffered saline (PBS) were intranasally administered at different time points $(1 \mathrm{~h}, 6 \mathrm{~h}$ or $12 \mathrm{~h})$ after the surgery for three consecutive days. ERK $1 / 2$ and PKC $\beta$ activators, Ceramide C6 (5mg/kg, sc-3527, Santa Cruz, USA) and Phorbol 12-myristate 13-acetate (PMA) (480 pmol/pup, ab120297, Abcam, USA), were dissolved in 5\% dimethyl sulfoxide (DMSO), after which $3 \mathrm{ul}$ was administered intracerebroventricularly at $24 \mathrm{~h}$ before $\mathrm{GMH}$ or sham surgery.

\subsection{Neurobehavioral assessment}

Negative geotaxis and righting reflex were employed to exam the short-term neurobehavior while water maze, foot fault and rotarod tests were used for long-term neurobehavioral assessment as previously reported from our laboratory $[8,37]$. All of the tests were executed by two researchers who were double blinded to the experimental groups. 


\subsubsection{Negative Geotaxis}

A rough ramp was adjusted to an angle of 45 degrees. Experimental pups were put on it in a downward orientation and they were expected to adjust to an upward position. The time it took the pups to correct their bodies parallel to the ground plane (90 degrees) and upside down (180 degrees) were recorded. 60 seconds was recorded when pups rolled from ramp or corrected time was over 60 seconds. Each test was repeated three times and the average result of three consecutive days was taken for statistical analysis.

\subsubsection{Righting Reflex}

Pups were placed supinely on a horizontal plate and the time of automatic rollover was recorded within 20 seconds. Every pup was also examined three times as in negative geotaxis.

\subsubsection{Morris Water Maze}

Morris water maze was performed to evaluate cognitive function between 22 and 27 days post hemorrhage as previously described[38,39]. Briefly, after the training block, juvenile rats were placed in a round pool (1.10 $\mathrm{m}$ in diameter) filled with suitable temperature and opacity water, in which a platform $(10 \mathrm{~cm}$ in diameter) was located at $1 \mathrm{~cm}$ below the water level. The time and distance it took for the animals to find platform were recorded over four blocks. Then the platform was removed. A probe trial was performed to measure the percentage of swimming time in the quadrant where the platform was located.

\subsubsection{Rotarod and foot fault test}

Rotarod and foot fault tests were conducted to detect the motor and proprioception function post hemorrhage as previously described $[9,40]$. After training, juvenile rats were placed on a rotor, which is 70 $\mathrm{mm}$ in diameter, and they must walk forward to maintain their balance. The test started at 5 RPM or 10 RPM and increases by 2 RPM every 5 seconds. The time before the rats fell was recorded by the machine (Columbus Instruments, USA). For the foot fault test, juvenile rats were placed on a shelf with wire grills $(0.6 \times 1.5 \mathrm{~m}, 2.5 \mathrm{~cm}$ apart$)$ to move freely and the numbers of missteps were recorded within 1 minute.

\subsection{Western bolt}

The experimental rats' forebrain samples were extracted and the target proteins were detected by Western Blot as previously described[41]. Briefly, both hemispheres were homogenized in RIPA lysis buffer (sc24948, Santa Cruz, USA) and then concentrated at 14 000rpm for 30 minutes. The supernatant was extracted and the total protein concentration was calculated using spectrophotometer (Bio-Rad). Protein samples ( $4 \mathrm{uL}, 1.5 \mathrm{mg} / \mathrm{mL}$ ) were loaded on $7.5-15 \%$ tris-glycine gel, electrophoresed, and transferred to 0.2 or 0.45 um nitrocellulose membranes. Membranes were incubated overnight at $4^{\circ} \mathrm{C}$ with the primary antibodies: rabbit polyclonal anti-IL-17A (1:500, GTX32674; Gene Tex), rabbit polyclonal anti-IL-17RA antibody (1:500, ab180904; Abcam), rabbit polyclonal anti-PKC $\beta 1+P K C \beta 2$ antibody (1:1000, ab194749; Abcam), rabbit monoclonal anti-phosphorylated ERK (1:1000, Santa Cruz); mouse monoclonal anti-ERK (1:2000, sc-514302, Santa Cruz); rabbit polyclonal anti-phosphorylated NFkB (1:1000, ab76302, Abcam), 
rabbit polyclonal anti-NF-KB (1:500, ab140751, Abcam); rabbit polyclonal anti-TNF-a (1:1000, ab6671, Abcam), rabbit monoclonal anti-IL-1 3 (1:2000, ab205924, Abcam); rabbit polyclonal anti-IL-6 (1:5000, ab208113, Abcam); mouse anti- $\beta$-actin (1:2000, sc-47778, Santa Cruz). The membrane was then exposed to radiography films to display the protein bands. The density of bands was analyzed for the relative density of the resultant protein immunoblot by ImageJ software $(\mathrm{NIH})$.

\subsection{Immunofluoscence}

Rat pups were anesthetized by isoflurane which was followed by transcardiac perfusion with $4^{\circ} \mathrm{C}$ PBS and $4 \%$ paraformaldehyde. The whole brain was removed for cryopreservation and cut into $5 \mu \mathrm{m}$ coronal sections equidistantly. Immunofluorescence staining was performed as previously described[42]. Sections were stained with primary antibodies of IL-17RA (1:200, ab180904, Abcam), IL-1ß (1:500, ab9722, Abcam), myeloperoxidase (MPO) (1:500, ab65871, Abcam), Iba-1(1:100, ab48004, Abcam) at $4^{\circ} \mathrm{C}$ overnight. They then were incubated with the appropriate fluorescence-conjugated secondary antibodies (1:200, Jackson ImmunoResearch Labs) for 1 hour at room temperature followed by mounting media with DAPI. The peri-hemorrhagic area was imaged by a DMi8 fluorescent microscope (Leica Microsystems) under a $400 \times$ fold field. To detect the IL-1 $\beta$, MPO and Iba- 1 positive cells, four fields from the perihematomal region were taken, while the same visual fields were used in sham group.

\subsection{Statistical analysis}

Sample size evaluation was determined using a type I error rate of 0.05 and a power of 0.8 on a 2-sided test by power analysis. Parametric data was described as mean \pm SD and analyzed using one-way ANOVA followed by Tukey's post-hoc test. Longitudinal data were analyzed using two-way repeated measure ANOVA with Tukey's post-hoc test. $P$ values of $<0.05$ were considered statistically significant. GraphPad Prism 7 (La Jolla) were used for graphing and analyzing all the data.

\section{Result}

\subsection{Endogenous expression of IL-17A, IL-17RA, PKC $\beta$, P- ERK increased after GMH}

The endogenous proteins were estimated by Western blot at 0 (sham), 6h, 12h, 24h, 3d and 7d post-GMH. Endogenous IL-17 expression significantly increased from 24 hours to 7 days and peaked at 24 hours after GMH (Figure 2, A and B). IL-17RA expression increased and the trend was similar to its ligand (Figure 2, A and C). Both PKC $\beta$ and p-ERK expression increased simultaneously after GMH (Figure 2, A, D and E), while IL-20R2 increased from 12 hours to 7 days and peaked at 5 days after GMH (Figure 2, A and D). Based on these results, the $24 \mathrm{~h}$ time point was chosen to study the mechanism of action.

\subsection{IL-17RA is expressed on microglia both in sham and GMH pups.}


Double immunofluorescence staining was used to determine the cellular localization of IL-17RA on microglia (Iba-1) at 24 hours after GMH. The results indicated that IL-17RA was expressed on microglia in both sham and GMH animals (Figure 3).

\subsection{Intranasal administration of secukinumab improved short-term behavioral outcomes at $1 \mathrm{~h}$ and $6 \mathrm{~h}$ after $\mathrm{GMH}$ surgery.}

In a previous study, we confirmed the best dosage $(0.6 \mathrm{mg} / \mathrm{kg})$ for treatment of $\mathrm{GMH}[36]$. Three different time points $(1 \mathrm{~h}, 6 \mathrm{~h}, 12 \mathrm{~h})$ were chosen to optimize treatment. Hence, the following animal groups were used for the time-response study: Sham, GMH + vehicle, GMH + secukinumab $(0.6 \mathrm{mg} / \mathrm{kg}, 1 \mathrm{~h}), \mathrm{GMH}+$ secukinumab $(0.6 \mathrm{mg} / \mathrm{kg}, 6 \mathrm{~h})$, and $\mathrm{GMH}+$ secukinumab $(0.6 \mathrm{mg} / \mathrm{kg}, 12 \mathrm{~h})$. Compared to sham, each treatment group had significant short-term neurological impairment at 24h after GMH. At $48 \mathrm{~h}$ and $72 \mathrm{~h}$ after $\mathrm{GMH}$, the $1 \mathrm{~h}$ and $6 \mathrm{~h}$ time-point groups had statistically higher score in both negative geotaxis (90 and 180 degrees) and righting reflex tests (Figure 4, A-C). Additionally, at $72 \mathrm{~h}$ after GMH, the $12 \mathrm{~h}$ timepoint group had statistically higher score in righting reflex as well (Figure $4, C$ ). Based on the experimental evaluation results above, the $1 \mathrm{~h}$ time point treatment was selected for further research.

\subsection{IL-17A inhibition with secukinumab attenuated neuroinflammation at $24 \mathrm{~h}$ after $\mathrm{GMH}$.}

IL-1 $\beta$ and MPO were chosen as the indicators of neuroinflammation and immunofluorescence staining was used to count the numbers of microglia. At $24 \mathrm{~h}$ after $\mathrm{GMH}$, there were significantly increased microglia activation compared to sham animals. Expression of IL-1 $\beta$ and MPO had the same trend as Iba1 (Figure 5, A-F). However, immunofluorescence staining showed that secukinumab significantly reduced the numbers of Iba-1, IL-1 $\beta$, and MPO-positive cells in the perihematomal area compared to those in the $\mathrm{GMH}+$ vehicle group (Figure 5, A-F).

3.5 Secukinumab improved long-term behavioral outcomes while PMA or Ceramide C6 abolished its beneficial effects after $\mathrm{GMH}$.

To assess the effects of secukinumab on long-term neurological function (memory and motor skills), neurobehavioral tests were performed between 22nd and 28th days after $\mathrm{GMH}$. For six consecutive days, Morris water maze was performed to evaluate memory function starting at 22 days after $\mathrm{GMH}$. After training, the water maze test was performed every day over a period of four days (Day 1 to Day 4). Animals from different experimental groups began with comparable escape latency and swim distance on Day 1. On Day 2, we observed that all GMH animals, including vehicle and different treatment groups, showed more swim distance (Figure 6, A) and more escape latency (Figure 6, B). However, on Day 3 and Day 4, secukinumab treated group took significantly less time and distance to find the platform compared to the vehicle group (Figure 6A, B). However, the beneficial effect of treatment was abolished 
by PMA and Ceramide $\mathrm{C} 6$ interventions. There was no statistical difference in the averaged swimming speed of each group (data not shown). A Probe Trial following Day 4 showed that the vehicle-treated group spent significantly less time in the target quadrant compared to sham. PMA and ceramide C6 treated animals had similar outcomes compared to the vehicle group, while secukinumab treated animals spent more time in the target quadrant (Figure 6. G). Rotarod and foot fault tests were conducted to evaluate the motor ability on the 28th day after $\mathrm{GMH}$. Vehicle-treated animals had much shorter falling latency on both $5 \mathrm{rmp}$ and $10 \mathrm{rmp}$ accelerating rods compared to the sham animals. The secukinumabtreated group had shorter latency than the vehicle-treated group (Figure 6, C), and the improvement was abolished by the interventions of PMA and ceramide C6. Meanwhile, the number of total missteps from the vehicle-treated group within 1 minute was more than that of sham. Secukinumab treatment partially normalized the motor function (Figure 6, E). Yet, the same reversing effect was found with PMA and ceramide $\mathrm{C} 6$ treatments. Thus, secukinumab restored the motor function in $\mathrm{GMH}$ rats and the activators of PKC $\beta$ and $p$-ERK reversed this effect. There was no statistical difference observed between male and female rats (data not shown) in these tests.

\subsection{PKC $\beta$ activator PMA and p-ERK activator ceramide C6 abolished the anti-inflammatory effects of secukinumab.}

Secukinumab inhibition of IL-17A and its downstream factors, such as PKC $\beta$ and p-ERK, play a crucial role in expression of NF-KB that leads to suppressed neuroinflammation after GMH. Thus, we evaluated the effects of the treatments on the expression of IL-17RA, PKC $3, \mathrm{p}-\mathrm{ERK}, \mathrm{NF}-\mathrm{KB}$ and inflammation markers, IL-1 $\beta$, TNF-aand IL-6, at 24 hours post GMH (Figure 7). The expression of IL-17RA, PKC $\beta$, $p$-ERK, NF-KB, IL-1 $\beta$, TNF-aand IL-6 increased after GMH. Except for IL-17RA, all these protein expression levels decreased with secukinumab treatment. PMA significantly activated the expression of PKC $\beta$ at 24 hours after intranasal administration. Western blot outcomes also showed that PMA significantly increased phosphorylated ERK and the expression of NF-KB, which was accompanied by increase of inflammation factors, IL-1 $\beta$, TNF-aand IL-6, at 24 hours after GMH. Furthermore, the results demonstrated that the activator of $p$-ERK also abolished the effects of secukinumab on the $p$-ERK and inflammation factors in a similar manner to PMA (Figure 7). Secukinumab did not alter IL-17RA level. Neither did we observe any effect of ceramide $\mathrm{C6}$ on $\mathrm{PKC} \beta$.

\section{Discussion}

Germinal matrix hemorrhage is devastating disease for neonates with no noninvasive treatments. Inflammatory response contributes the brain injury after $\mathrm{GMH}$ and microglia play a critic role in mediating the process[9]. In this study, we first investigated the role of IL-17A inhibitor secukinumab in neuroinflammation after $\mathrm{GMH}$ in rat pups and the potential mechanism involving the IL17RA/PKC $\beta / E R K / N F-K B$ signaling pathway. The following outcomes were found. (a) Endogenous protein levels of PKC $\beta$ and $p$-ERK increased, which was accompanied by the increase of IL-17A and its receptor IL-17RA. (b) Intranasal administration of IL-17A antibody secukinumab at $1 \mathrm{~h}$ after GMH significantly 
improved short-term neurological outcomes; reduced the expression of IL-1 3, MPO and Iba-1 near the area of hematoma. (c) Secukinumab attenuated long-term neurobehavioral deficits after GMH. However, the respective activators of PKC $\beta$ and p-ERK, PMA and ceramide $\mathrm{C6}$, abolished the treatment effects of secukinumab. (d) Secukinumab suppressed the expression of inflammatory factors after GMH, while PMA and ceramide $\mathrm{C} 6$ offset this phenomenon.

The function of IL-17A in the inflammatory cascade has been confirmed by mounting evidence $[43,44]$. IL-17 is secreted mainly by immune cells, such as Th17 cells and $\gamma \delta T$ cells [45-47]. In the event of brain injuries, these immune cells can be activated through various upstream stimuli $[48,49]$. Hence, it is deducible that endogenous IL-17A would increase after GMH. Secukinumab, a recombinant monoclonal antibody for IL-17A, is used as an anti-inflammatory treatment for some severe chronic immune diseases, such as psoriasis [33, 34]. After $\mathrm{GMH}$, microglia and macrophages recruited from the blood accelerate the release of inflammatory mediators, which contribute to the impairment of neurological behavior. Consistent with the previous studies, our results showed that secukinumab decreased the expression of pro-inflammatory cytokine IL-1ßand MPO in perihematomal areas of GMH, inhibited the activation of microglia, and improved the short- and long-term neurobehavioral outcomes. Also, we observed that secukinumab attenuated the elevated expression of NF-KB, IL-1 $\beta$, TNF- $\alpha$ and IL- 6 in the hemorrhaged brain as shown by Western blot. Interestingly, we noticed that, only early administration of secukinumab (1h) could attenuate the defected of neurological function after GMH. This result suggested that early inflammation response plays a very important role in secondary brain injury after $\mathrm{GMH}$.

In CNS, IL-17RA is expressed on all kinds of resident cells, including microglia, astrocytes, neurons and endothelial cells $[43,50,51]$. IL-17RA signaling on microglia has been reported by many studies and became the target for drugs[43,52]. There was no intracellular enzymatic activity that has been found on IL-17RA and its complexes [53]. PKC $\beta$, one of the protein kinases, performs its function of controlling other proteins via phosphorylating hydroxyl groups of serine and threonine amino acid residues on these proteins[25]. Under the influence of PKC $\beta$ directly or indirectly, inactive ERK(1/2) changes into active pERK $[24,25,54]$, which translocates to the nucleus to trigger the expression of inflammatory activator NF$\kappa B[27,55]$. Meanwhile, $P K C \beta$ and $p$-ERK have been reported to be associated with neuroinflammation in microglia[56, 57].

In our research, we observed that IL-17A and its receptor increased along with PKC $\beta$ and p-ERK in the time course study. Also, the increased expression of PKC $\beta$ and p-ERK was attenuated by the treatment of IL17A inhibitor secukinumab after GMH. To further explore the possible mechanism underlying secukinumab mediated anti-inflammtory response after $\mathrm{GMH}, \mathrm{PKC} \beta$ and $\mathrm{p}$-ERK activator, PMA and ceramide $\mathrm{C6}$, have been chosen. After being administered with secukinumab, both activators abrogated the decreased expression of inflammatory cytokines, IL-1 $\beta$, TNF- $\alpha$ and IL-6. Meanwhile, consistent with the expression of these proteins, the same effects could be found on the long-term neurological behavior of juvenile rats after $\mathrm{GMH}$ as well. However, $\mathrm{p}$-ERK activator Ceramide $\mathrm{C} 6$ had no effect on the expression of PKC $\beta$. Therefore, we presumed $p$-ERK is the downstream protein of PKC $\beta$. Taken together, our data 
suggested that secukinumab exerted its protective effect at least partially through $\mathrm{PKC} \beta / \mathrm{p}-\mathrm{ERK} / \mathrm{NF}-\mathrm{KB}$ pathway.

Some limitations should not be ignored in this study and more unknown mechanisms need to be explored. First, brain injury has a very complex pathological process after $\mathrm{GMH}$. Except neuroinflammation, other pathological processes also take place, such as the impairment of blood-brain barrier integrity, autophagy and neurogenesis. It is not clear whether they are associated with IL-17A and its pathway. Second, there may be other downstream pathways existing and playing a potential role. Future studies are needed to fully elucidate the potential mechanisms associated with secukinumab in the setting of experimental GMH. Third, IL-17RA also expresses on other cells, including neurons, and its function need to be further investigated. Last, we have not elucidated whether the change in protein expression was attributed to genetic modification. Further studies on a molecular level would be needed.

\section{Conclusion}

In this current study, we demonstrated that secukinumab treatment attenuated neurological deficits and inhibited neuroinflammation after $\mathrm{GMH}$ in neonatal rat pups. The protective effects were mediated through the activation of $\mathrm{PKC} \beta / E R K / N F-K B$ signaling pathway. Our study is the first to demonstrate secukinumab effects on inhibition of neuroinflammation, providing new insight for non-invasive therapeutic strategies for the management of $\mathrm{GMH}$.

\section{Abbreviations}

CNS: central nervous system; DAPI: 4',6-diamidino-2-phenylindole; DMSO: dimethyl sulfoxide; ERK: extracellular signal-regulated kinase; p-ERK: Phosphorylated extracellular signal-regulated kinase; GMH: germinal matrix hemorrhage; IF: immunofluorescence; IL-17: interleukin-17; IL-17RA: interleukin-17A receptor; MPO: myeloperoxidase; NF-кB: nuclear factor-k-gene binding; p-NF-кB: phosphorylated nuclear factor-k-gene binding: PBS, phosphate-buffered saline; PCK 3 , protein kinase $C$ beta; PMA: phorbol 12myristate 13-acetate; Sec.: secukiunumab; WB: western blot.

\section{Declarations}

\section{Authors' contributions}

SPL and SXD participated in the research design, experimental performance (including animal surgery, Western blotting, and immunohistochemistry, but not the neurobehavioral testing), data analysis, and drafting of the manuscript. YD and JJF provided technical assistance and help with the manuscript preparation. XLZ and XJJ discussed the results and edited parts of the manuscript. XH, JP and GZ performed the intracerebroventricular injection, co-immunoprecipitation, behavioral tests, and data analysis. JJF and JHZ participated in the research design and edited the manuscript. YG and JPT are the 
corresponding authors; these authors participated in all aspects of the study, including research design, data analysis, and manuscript preparation. The authors read and approved the final manuscript.

\section{Availability of data and materials}

The datasets used and/or analyzed in the current study are available from

the corresponding authors on request.

\section{Acknowledgements}

Not applicable.

\section{Ethics approval and consent to participate}

All animal experimental protocols were approved by the Loma Linda

University Animal Care and Use Committees (IACUCs).

\section{Consent for publication}

Not applicable.

\section{Competing interests}

The authors declare that they have no competing interests.

\section{Funding}

This study is supported partially by grants from National Institutes of Health (NS082184 to John Zhang, NS101284 to Jiping Tang) and by grants from the Science and Technology Commission of Shanghai Municipal (21ZR1410700 to S.D.).

\section{Consent for publication}

Not applicable.

\section{Author details}

Shengpeng Liu ${ }^{1,3+}$, Shuixiang Deng ${ }^{2,3+}{ }^{\dagger}$, Yan Ding ${ }^{3}$, Jerry J. Flores ${ }^{3}$, Xiaoli Zhang ${ }^{1}$, Xiaojing Jia ${ }^{1}$, Xiao Hu ${ }^{3}$, Jun Peng ${ }^{3}$, Gang Zuo ${ }^{3}$, John H. Zhang ${ }^{3,4,5}$, Ye Gong $^{2,6^{*}}$, and Jiping Tang ${ }^{3^{*}}$

${ }^{1}$ Department of Pediatrics, Shenzhen People's Hospital (The Second Clinical Medical College; Jinan University; The First Affiliated Hospital, Southern University of Science and Technology), Shenzhen 518020, Guangdong, China 
${ }^{2}$ Department of Critical Care Medicine, HuaShan Hospital, Fudan University, Shanghai, 200040, China

${ }^{3}$ Department of Physiology and Pharmacology, Center for Neuroscience Research, Loma Linda University School of Medicine, Loma Linda, CA 92350, USA

${ }^{4}$ Department of Neurosurgery, Loma Linda University School of Medicine, Loma Linda, CA, 92350, USA

${ }^{5}$ Department of Anesthesiology, Loma Linda University School of Medicine, Loma Linda, CA 92350, USA

${ }^{6}$ Department of Neurosurgery, Huashan Hospital, Fudan University, Shanghai, 200040, China.

\section{References}

1. Tortora D, Severino M, Malova M, Parodi A, Morana G, Sedlacik J, Govaert P, Volpe J, Rossi A, Ramenghi L: Differences in subependymal vein anatomy may predispose preterm infants to GMH-IVH. Archives of disease in childhood Fetal and neonatal edition 2018, 103:F59-F65.

2. Ramenghi L, Fumagalli M, Groppo M, Consonni D, Gatti L, Bertazzi P, Mannucci P, Mosca F: Germinal matrix hemorrhage: intraventricular hemorrhage in very-low-birth-weight infants: the independent role of inherited thrombophilia. Stroke 2011, 42:1889-1893.

3. Flores J, Klebe D, Tang J, Zhang J: A comprehensive review of therapeutic targets that induce microglia/macrophage-mediated hematoma resolution after germinal matrix hemorrhage. Journal of neuroscience research 2020, 98:121-128.

4. Xu M, Seyler L, Bäuerle T, Kalinichenko L, Müller C, Huttner H, Schwab S, Manaenko A: Serelaxin activates eNOS, suppresses inflammation, attenuates developmental delay and improves cognitive functions of neonatal rats after germinal matrix hemorrhage. Scientific reports 2020, 10:8115.

5. Tang J, Tao Y, Tan L, Yang L, Niu Y, Chen Q, Yang Y, Feng H, Chen Z, Zhu G: Cannabinoid receptor 2 attenuates microglial accumulation and brain injury following germinal matrix hemorrhage via ERK dephosphorylation in vivo and in vitro. Neuropharmacology 2015, 95:424-433.

6. Koschnitzky J, Keep R, Limbrick D, McAllister J, Morris J, Strahle J, Yung Y: Opportunities in posthemorrhagic hydrocephalus research: outcomes of the Hydrocephalus Association Posthemorrhagic Hydrocephalus Workshop. Fluids and barriers of the CNS 2018, 15:11.

7. Fowlie P, Davis P: Prophylactic indomethacin for preterm infants: a systematic review and metaanalysis. Archives of disease in childhood Fetal and neonatal edition 2003, 88:F464-466.

8. Li P, Zhao G, Ding Y, Wang T, Flores J, Ocak U, Wu P, Zhang T, Mo J, Zhang J, Tang J: Rh-IFN-a attenuates neuroinflammation and improves neurological function by inhibiting NF-KB through JAK1- 
STAT1/TRAF3 pathway in an experimental GMH rat model. Brain, behavior, and immunity 2019, 79:174185.

9. Zhang Y, Xu N, Ding Y, Zhang Y, Li Q, Flores J, Haghighiabyaneh M, Doycheva D, Tang J, Zhang J: Chemerin suppresses neuroinflammation and improves neurological recovery via CaMKK2/AMPK/Nrf2 pathway after germinal matrix hemorrhage in neonatal rats. Brain, behavior, and immunity 2018, 70:179193.

10. Feng Z, Ye L, Klebe D, Ding Y, Guo Z, Flores J, Yin C, Tang J, Zhang J: Anti-inflammation conferred by stimulation of CD200R1 via Dok1 pathway in rat microglia after germinal matrix hemorrhage. Journal of cerebral blood flow and metabolism : official journal of the International Society of Cerebral Blood Flow and Metabolism 2019, 39:97-107.

11. Supramaniam V, Vontell R, Srinivasan L, Wyatt-Ashmead J, Hagberg H, Rutherford M: Microglia activation in the extremely preterm human brain. Pediatric research 2013, 73:301-309.

12. Aronoff D, Carstens J, Chen G, Toews G, Peters-Golden M: Short communication: differences between macrophages and dendritic cells in the cyclic AMP-dependent regulation of lipopolysaccharideinduced cytokine and chemokine synthesis. Journal of interferon \& cytokine research : the official journal of the International Society for Interferon and Cytokine Research 2006, 26:827-833.

13. Tang J, Chen Q, Guo J, Yang L, Tao Y, Li L, Miao H, Feng H, Chen Z, Zhu G: Minocycline Attenuates Neonatal Germinal-Matrix-Hemorrhage-Induced Neuroinflammation and Brain Edema by Activating Cannabinoid Receptor 2. Molecular neurobiology 2016, 53:1935-1948.

14. Xu P, Hong Y, Xie Y, Yuan K, Li J, Sun R, Zhang X, Shi X, Li R, Wu J, et al: TREM-1 Exacerbates Neuroinflammatory Injury via NLRP3 Inflammasome-Mediated Pyroptosis in Experimental Subarachnoid Hemorrhage. Translational stroke research 2020.

15. Brouwer $M$, de Vries L, Kersbergen $K$, van der Aa N, Brouwer A, Viergever M, Išgum I, Han K, Groenendaal F, Benders M: Effects of Posthemorrhagic Ventricular Dilatation in the Preterm Infant on Brain Volumes and White Matter Diffusion Variables at Term-Equivalent Age. The Journal of pediatrics 2016, 168:41-49.e41.

16. Zeng H, Fu X, Cai J, Sun C, Yu M, Peng Y, Zhuang J, Chen J, Chen H, Yu Q, et al: Neutrophil Extracellular Traps may be a Potential Target for Treating Early Brain Injury in Subarachnoid Hemorrhage. Translational stroke research 2021.

17. Zhang Q, Liao Y, Liu Z, Dai Y, Li Y, Li Y, Tang Y: Interleukin-17 and ischaemic stroke. Immunology 2020.

18. Lin Y, Zhang J, Yao C, Wu Y, Abdelgawad A, Yao S, Yuan S: Critical role of astrocytic interleukin-17 A in post-stroke survival and neuronal differentiation of neural precursor cells in adult mice. Cell death \& 
disease 2016, 7:e2273.

19. Park M, Pilla R, Panta A, Pandey S, Sarawichitr B, Suchodolski J, Sohrabji F: Reproductive Senescence and Ischemic Stroke Remodel the Gut Microbiome and Modulate the Effects of Estrogen Treatment in Female Rats. Translational stroke research 2020, 11:812-830.

20. Shi H, Wang J, Wang J, Huang Z, Yang Z: IL-17A induces autophagy and promotes microglial neuroinflammation through ATG5 and ATG7 in intracerebral hemorrhage. Journal of neuroimmunology 2018, 323:143-151.

21. Yang Z, Liu Q, Shi H, Jiang X, Wang S, Lu Y, Zhang J, Huang X, Yu A: Interleukin 17A exacerbates ER-stress-mediated inflammation of macrophages following ICH. Molecular immunology 2018, 101:3845 .

22. Gao L, Li P, Shao T, Mao X, Qi H, Wu B, Shan M, Ye L, Cheng H: Neurotoxic role of interleukin-17 in neural stem cell differentiation after intracerebral hemorrhage. Neural regeneration research 2020, 15:1350-1359.

23. Liu Y, Zhu H, Su Z, Sun C, Yin J, Yuan H, Sandoghchian S, Jiao Z, Wang S, Xu H: IL-17 contributes to cardiac fibrosis following experimental autoimmune myocarditis by a PKC $\beta /$ Erk1/2/NF-KB-dependent signaling pathway. International immunology 2012, 24:605-612.

24. Bowen C, Calderón Giadrosic J, Burger Z, Rykiel G, Davis E, Helmers M, Benke K, Gallo MacFarlane E, Dietz H: Targetable cellular signaling events mediate vascular pathology in vascular Ehlers-Danlos syndrome. The Journal of clinical investigation 2020, 130:686-698.

25. Luan Y, Zhang J, Wang M, Fu G, Zhang W: Advanced glycation end products facilitate the proliferation and reduce early apoptosis of cardiac microvascular endothelial cells via PKC $\beta$ signaling pathway: Insight from diabetic cardiomyopathy. Anatolian journal of cardiology 2020, 23:141-150.

26. Zhu Y, Tan Y, Leung L: Aflatoxin B1 disrupts transient receptor potential channel activity and increases COX-2 expression in JEG-3 placental cells. Chemico-biological interactions 2016, 260:84-90.

27. Ala M, Mohammad Jafari R, Ala M, Agbele A, Hejazi S, Tavangar S, Mahdavi S, Dehpour A:

Sumatriptan alleviates radiation-induced oral mucositis in rats by inhibition of NF-kB and ERK activation, prevention of TNF- $a$ and ROS release. Archives of oral biology 2020, 119:104919.

28. Ding X, Sun X, Shen X, Lu Y, Wang J, Sun Z, Miao C, Chen J: Propofol attenuates TNF-a-induced MMP-9 expression in human cerebral microvascular endothelial cells by inhibiting Ca/CAMK II/ERK/NFKB signaling pathway. Acta pharmacologica Sinica 2019, 40:1303-1313.

29. Durocher M, Knepp B, Yee A, Jickling G, Rodriguez F, Ng K, Zhan X, Hamade F, Ferino E, Amini H, et al: Molecular Correlates of Hemorrhage and Edema Volumes Following Human Intracerebral Hemorrhage 
Implicate Inflammation, Autophagy, mRNA Splicing, and T Cell Receptor Signaling. Translational stroke research 2020.

30. Serra D, Henriques J, Serra T, Bento Silva A, Bronze M, Dinis T, Almeida L: An Anthocyanin-Rich Extract Obtained from Portuguese Blueberries Maintains Its Efficacy in Reducing Microglia-Driven Neuroinflammation after Simulated Digestion. Nutrients 2020, 12.

31. Wang Z, Chen Q, Wang J, Yu L, Chen L: Sulforaphane mitigates LPS-induced neuroinflammation through modulation of Cezanne/NF-KB signalling. Life sciences 2020, 262:118519.

32. Polat Ekinci A, Bölük K, Kobaner G: Secukinumab and acitretin as a combination therapy for three clinical forms of severe psoriasis in multi-drug refractory patients: A case series of high efficacy and safety profile. Dermatologic therapy 2020:e14704.

33. Frieder J, Kivelevitch D, Menter A: Secukinumab: a review of the anti-IL-17A biologic for the treatment of psoriasis. Therapeutic advances in chronic disease 2018, 9:5-21.

34. Mease P, Mclnnes I, Kirkham B, Kavanaugh A, Rahman P, van der Heijde D, Landewé R, Nash P, Pricop L, Yuan J, et al: Secukinumab Inhibition of Interleukin-17A in Patients with Psoriatic Arthritis. The New England journal of medicine 2015, 373:1329-1339.

35. Lekic T, Manaenko A, Rolland W, Krafft P, Peters R, Hartman R, Altay O, Tang J, Zhang J: Rodent neonatal germinal matrix hemorrhage mimics the human brain injury, neurological consequences, and post-hemorrhagic hydrocephalus. Experimental neurology 2012, 236:69-78.

36. Liu S, Huang L, Flores J, Ding Y, Li P, Peng J, Zuo G, Zhang J, Lu J, Tang J: Secukinumab attenuates reactive astrogliosis via IL-17RA/(C/EBPB)/SIRT1 pathway in a rat model of germinal matrix hemorrhage. CNS neuroscience \& therapeutics 2019, 25:1151-1161.

37. Wang T, Zhang J, Li P, Ding Y, Tang J, Chen G, Zhang J: NT-4 attenuates neuroinflammation via TrkB/PI3K/Fox01 pathway after germinal matrix hemorrhage in neonatal rats. Journal of neuroinflammation 2020, 17:158.

38. Ohtomo R, Kinoshita K, Ohtomo G, Takase H, Hamanaka G, Washida K, Islam M, Wrann C, Katsuki $\mathrm{H}$, Iwata A, et al: Treadmill Exercise Suppresses Cognitive Decline and Increases White Matter Oligodendrocyte Precursor Cells in a Mouse Model of Prolonged Cerebral Hypoperfusion. Translational stroke research 2020, 11:496-502.

39. Jackson L, Dong G, Althomali W, Sayed M, Eldahshan W, Baban B, Johnson M, Filosa J, Fagan S, Ergul A: Delayed Administration of Angiotensin II Type 2 Receptor (AT2R) Agonist Compound 21 Prevents the Development of Post-stroke Cognitive Impairment in Diabetes Through the Modulation of Microglia Polarization. Translational stroke research 2020, 11:762-775. 
40. Tanaka M, Ogaeri T, Samsonov M, Sokabe M: The 5a-Reductase Inhibitor Finasteride Exerts Neuroprotection Against Ischemic Brain Injury in Aged Male Rats. Translational stroke research 2019, 10:67-77.

41. Li Q, Ding Y, Krafft P, Wan W, Yan F, Wu G, Zhang Y, Zhan Q, Zhang J: Targeting Germinal Matrix Hemorrhage-Induced Overexpression of Sodium-Coupled Bicarbonate Exchanger Reduces Posthemorrhagic Hydrocephalus Formation in Neonatal Rats. Journal of the American Heart Association 2018, 7.

42. Ding Y, Zhang T, Wu G, McBride D, Xu N, Klebe D, Zhang Y, Li Q, Tang J, Zhang J: Astrogliosis inhibition attenuates hydrocephalus by increasing cerebrospinal fluid reabsorption through the glymphatic system after germinal matrix hemorrhage. Experimental neurology 2019, 320:113003.

43. Das Sarma J, Ciric B, Marek R, Sadhukhan S, Caruso M, Shafagh J, Fitzgerald D, Shindler K, Rostami A: Functional interleukin-17 receptor $A$ is expressed in central nervous system glia and upregulated in experimental autoimmune encephalomyelitis. Journal of neuroinflammation 2009, 6:14.

44. Xu D, Robinson A, Ishii T, Duncan D, Alden T, Goings G, Ifergan I, Podojil J, Penaloza-MacMaster P, Kearney $\mathrm{J}$, et al: Peripherally derived $\mathrm{T}$ regulatory and $\mathrm{\delta} \delta \mathrm{T}$ cells have opposing roles in the pathogenesis of intractable pediatric epilepsy. The Journal of experimental medicine 2018, 215:1169-1186.

45. Moser T, Akgün $\mathrm{K}$, Proschmann U, Sellner J, Ziemssen T: The role of TH17 cells in multiple sclerosis: Therapeutic implications. Autoimmunity reviews 2020, 19:102647.

46. Ribeiro M, Brigas H, Temido-Ferreira M, Pousinha P, Regen T, Santa C, Coelho J, Marques-Morgado I, Valente C, Omenetti S, et al: Meningeal yठ T cell-derived IL-17 controls synaptic plasticity and short-term memory. Science immunology 2019, 4.

47. Waisman A, Hauptmann J, Regen T: The role of IL-17 in CNS diseases. Acta neuropathologica 2015, 129:625-637.

48. Li S, Dai Q, Yu J, Liu T, Liu S, Ma L, Zhang Y, Han S, Li J: Identification of IL-17A-derived neural cell type and dynamic changes of IL-17A in serum/CSF of mice with ischemic stroke. Neurological research 2017, 39:552-558.

49. Zhong Q, Zhou K, Liang Q, Lin S, Wang Y, Xiong X, Meng Z, Zhao T, Zhu W, Yang Y, et al: Interleukin23 Secreted by Activated Macrophages Drives $Y \delta T$ Cell Production of Interleukin-17 to Aggravate Secondary Injury After Intracerebral Hemorrhage. Journal of the American Heart Association 2016, 5.

50. Li Z, Li K, Zhu L, Kan Q, Yan Y, Kumar P, Xu H, Rostami A, Zhang G: Inhibitory effect of IL-17 on neural stem cell proliferation and neural cell differentiation. BMC immunology 2013, 14:20.

51. Abdel-Maged A, Gad A, Rashed L, Azab S, Mohamed E, Awad A: Repurposing of Secukinumab as Neuroprotective in Cuprizone-Induced Multiple Sclerosis Experimental Model via Inhibition of Oxidative, 
Inflammatory, and Neurodegenerative Signaling. Molecular neurobiology 2020, 57:3291-3306.

52. Lin W, Wang N, Zhou K, Su F, Jiang Y, Shou J, Liu H, Ma C, Qian Y, Wang K, Wang X: RKIP mediates autoimmune inflammation by positively regulating IL-17R signaling. EMBO reports 2018, 19.

53. Santibanez J, Bjelica S: Novel Patents Targeting Interleukin-17A; Implications in Cancer and Inflammation. Recent patents on anti-cancer drug discovery 2018, 13:133-144.

54. Huang W, Mehta K: Modulation of Hepatic Protein Kinase C $\beta$ Expression in Metabolic Adaptation to a Lithogenic Diet. Cellular and molecular gastroenterology and hepatology 2015, 1:395-405.

55. Yang W, Kim J, Jeong D, Hong Y, Park S, Yang Y, Jang Y, Kim J, Cho J: 3-Deazaadenosine, an Sadenosylhomocysteine hydrolase inhibitor, attenuates lipopolysaccharide-induced inflammatory responses via inhibition of AP-1 and NF-kB signaling. Biochemical pharmacology 2020, 182:114264.

56. Sunohara J, Ridgway N, Cook H, Byers D: Regulation of MARCKS and MARCKS-related protein expression in BV-2 microglial cells in response to lipopolysaccharide. Journal of neurochemistry 2001, 78:664-672.

57. Yan J, Zuo G, Sherchan P, Huang L, Ocak U, Xu W, Travis Z, Wang W, Zhang J, Tang J: CCR1 Activation Promotes Neuroinflammation Through CCR1/TPR1/ERK1/2 Signaling Pathway After Intracerebral Hemorrhage in Mice.Neurotherapeutics : the journal of the American Society for Experimental NeuroTherapeutics 2020, 17:1170-1183.

\section{Figures}


Experiment 1: Time course of IL-17A, IL-17RA, PKC $\beta, p$-ERK and cellular localization of IL17RA after $\mathrm{GMH}$.

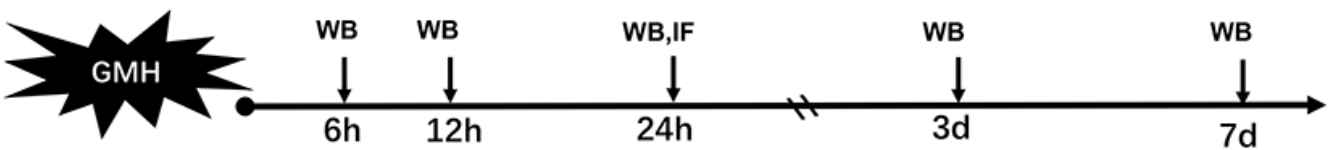

Experiment 2: The effects of secukinumab on short- and long-term neurobehavior.

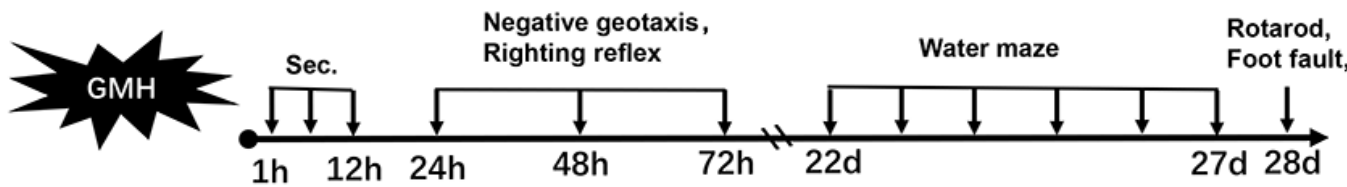

Experiment 3: The effects of secukinumab treatment on immunofluorescence expression of IL-1ß, MPO and Iba-1.

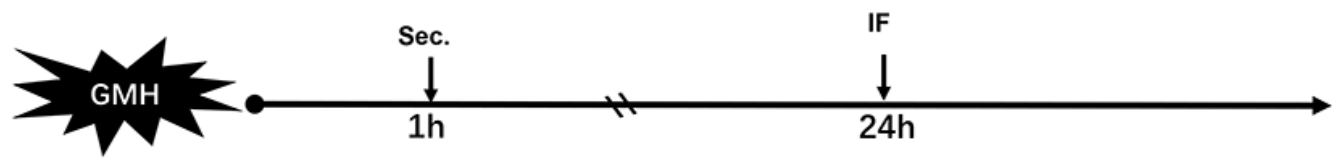

Experiment 4: The effects of PMA and Ceramide $\mathrm{C} 6$ on the treatment of secukinumab.

PMA, DMSO,

Ceramide C6,

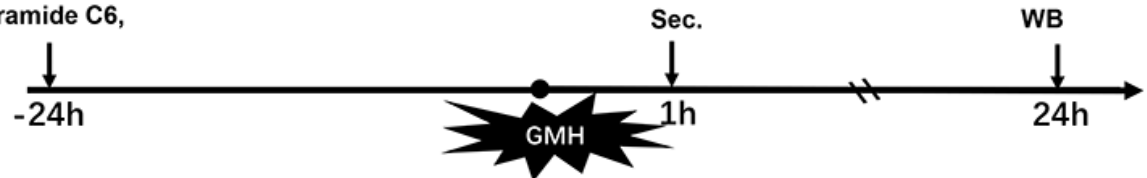

Groups:

(1)Sham

(2) GMH 6h

$(n=8)$ *

(3) GMH $12 \mathrm{~h}$

(4) GMH 24h

(5)GMH 3d

(6) GMH 7d

Groups (short-term, long-term):

(1)Sham $\quad(n=6,6)$

(2) GMH+vehicle $\quad(n=6,6)$

(3) GMH+Sec. $(0.6 \mathrm{mg} / \mathrm{kg}, 1 \mathrm{~h}) \quad(\mathrm{n}=6,6)$ **

(4) GMH+Sec. $(0.6 \mathrm{mg} / \mathrm{kg}, 6 \mathrm{~h}) \quad(n=6,0)$

(5) $\mathrm{GMH}+$ Sec. $(0.6 \mathrm{mg} / \mathrm{kg}, 12) \quad(\mathrm{n}=6,0)$

(6) $\mathrm{GMH}+$ Sec. $+\mathrm{PMA}$

$(n=0,6)$

(7) $\mathrm{GMH}+$ Sec.+Ceramide $\mathrm{C} 6$

$(n=0,6)$

Groups (IL-1 $\beta$, MPO , Iba-1):

(1)Sham

$(n=6)$

(2) GMH+vehicle

(3) $\mathrm{GMH}+$ Sec.

Groups:

(1)sham

(2) $\mathrm{GMH}+$ vehicle

(3) $\mathrm{GMH}+\mathrm{Sec}$.

(4) $\mathrm{GMH}+$ Sec. + PMA

(5) $\mathrm{GMH}+$ Sec. +DMSO

(6) $\mathrm{GMH}+$ Sec. + Ceramide $\mathrm{C} 6$

$(n=6)$

\section{Figure 1}

Experimental design and animal groups. * Extra 2 pups were used for IF (IL-17RA and Iba-1) at 24 hours after $\mathrm{GMH} .{ }^{* *}$ Assumed the optimal time group.

A

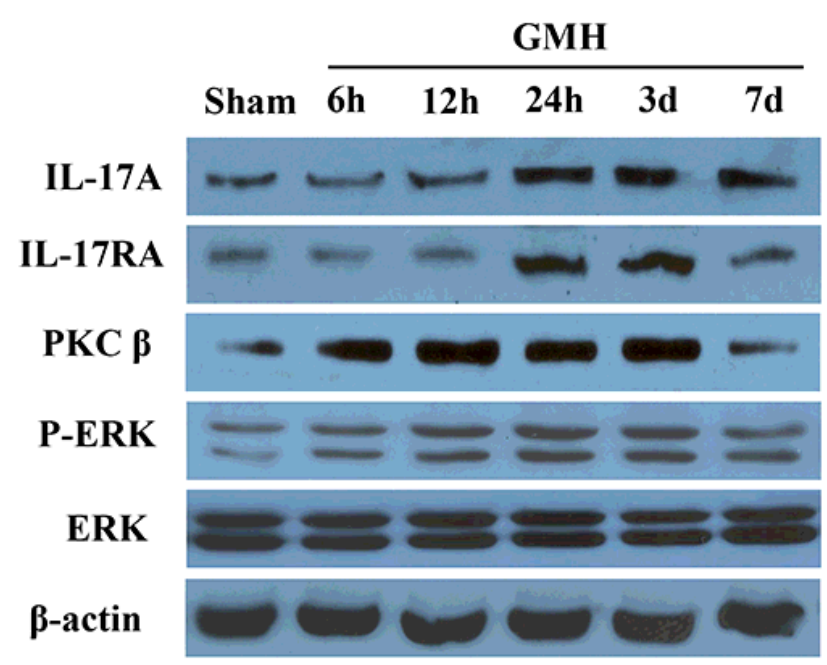

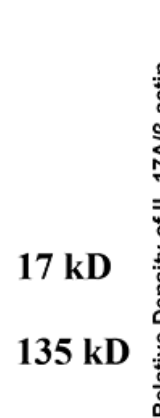

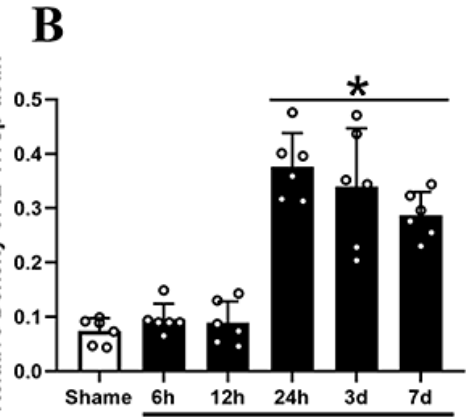

77 kD

$44 \mathrm{kD}$
$42 \mathrm{kD}$
$44 \mathrm{kD}$
$42 \mathrm{kD}$
$43 \mathrm{kD}$
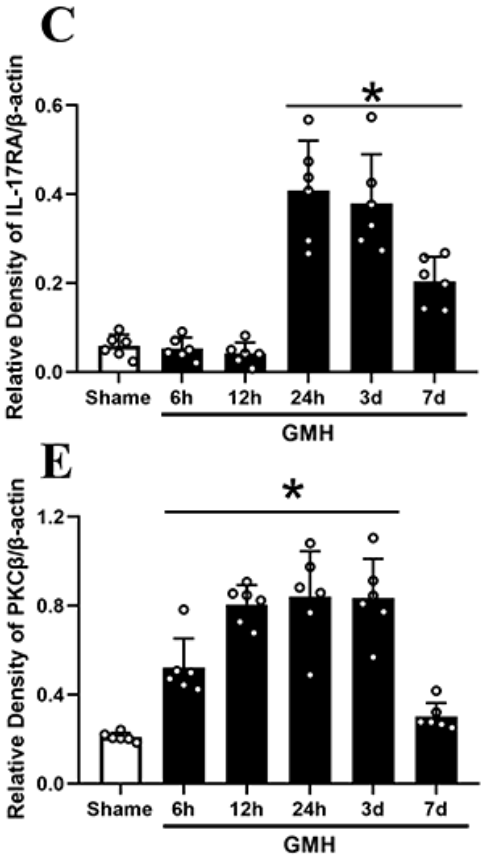

Figure 2 
The time course of endogenous levels of IL-17, IL-17RA, PKC $\beta$, and p-ERK after GMH. (A) Representative bands of IL-17, IL-17RA, PKC $\beta$, p-ERK, ERK and $\beta$-actin. (B) The expression of IL-17 at various time points. (C) The expression of IL-17RA at different time-points after GMH. (D) The expression of p-ERK at different time points after $\mathrm{GMH}$. (E) The expression of $\mathrm{PKC} \beta$ at different time points after $\mathrm{GMH}$. ${ }^{*} \mathrm{P}<0.05 \mathrm{vs}$. sham, mean $\pm S D$, one-way ANOVA, Tukey's test, $n=6 /$ group.
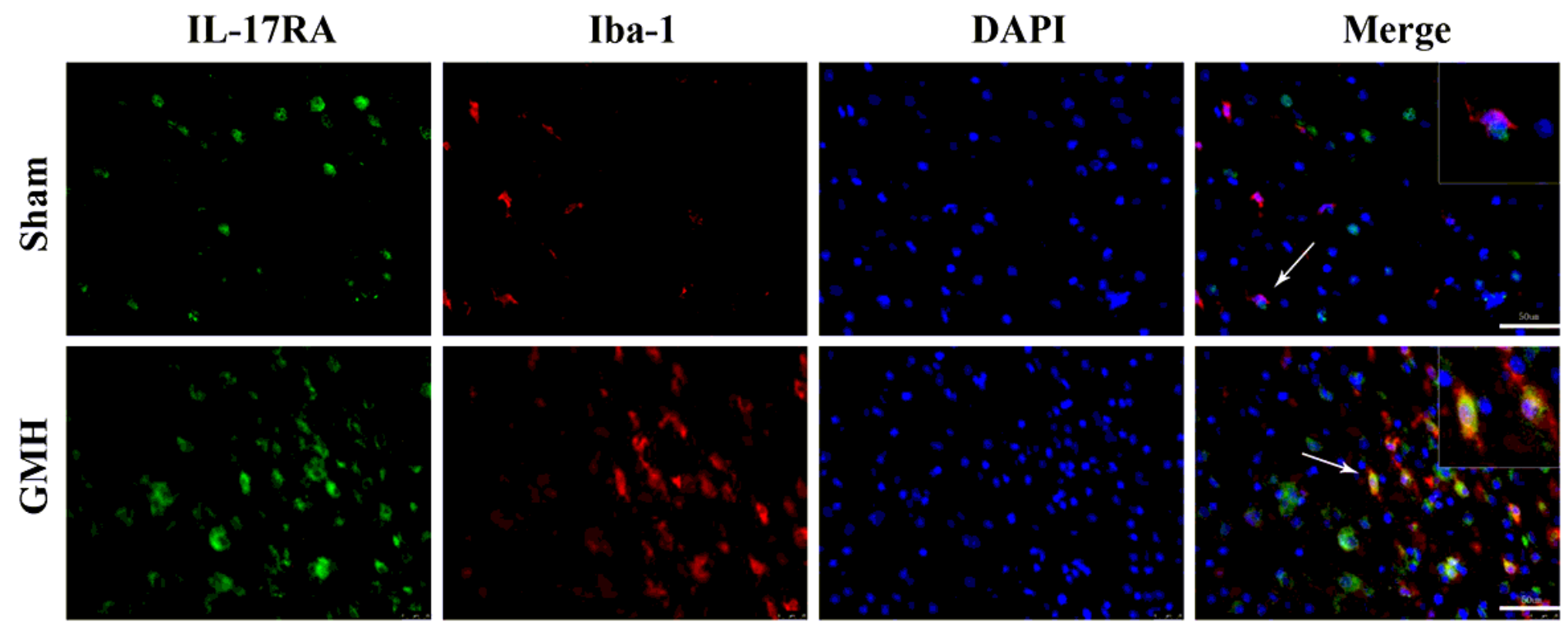

\section{Figure 3}

Colocalization of IL-17RA with Iba-1 at 24 hours after GMH. Scale bar=50 $\mu \mathrm{m}, \mathrm{n}=2 /$ group. 


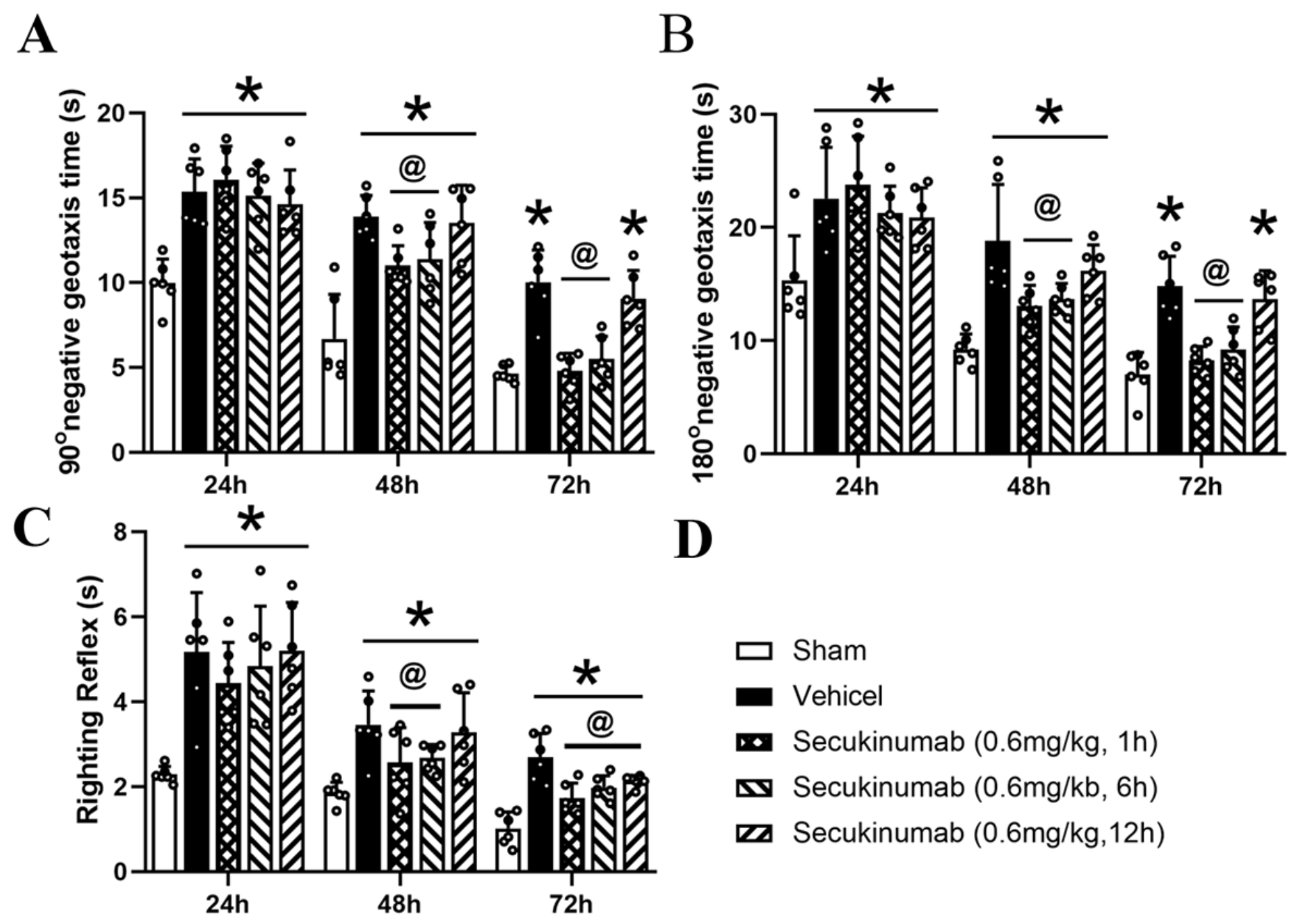

Figure 4

Intranasal administration of secukinumab improved short-term neurological function at 24, 48 and 72 hours after $\mathrm{GMH}$. (A) $90^{\circ}$ negative geotaxis; (B) $180^{\circ}$ negative geotaxis; (C) righting reflex. ${ }^{*} \mathrm{P}<0.05$ vs. Sham, @P $<0.05$ vs. GMH + Vehicle, mean \pm SD, two-way repeated measurement ANOVA, Tukey's test, $\mathrm{n}=8 /$ group. 

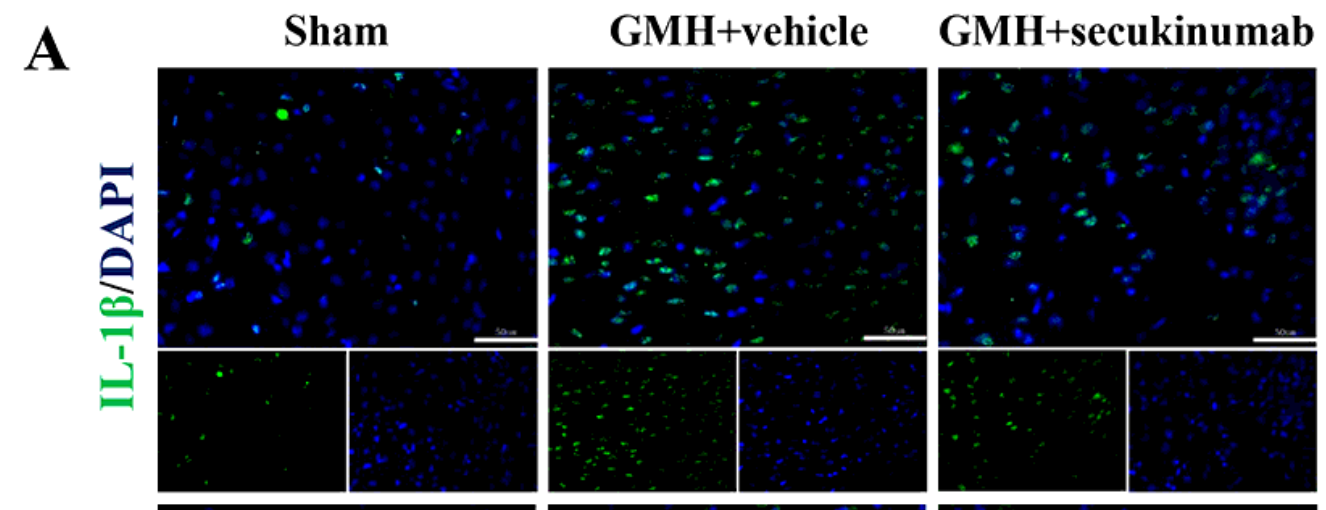

D
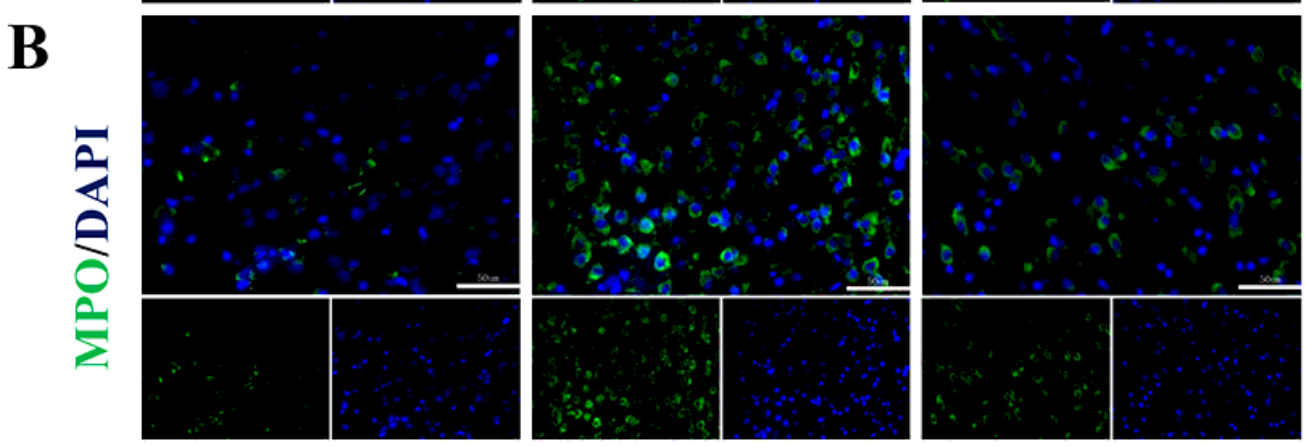

$\mathbf{E}$
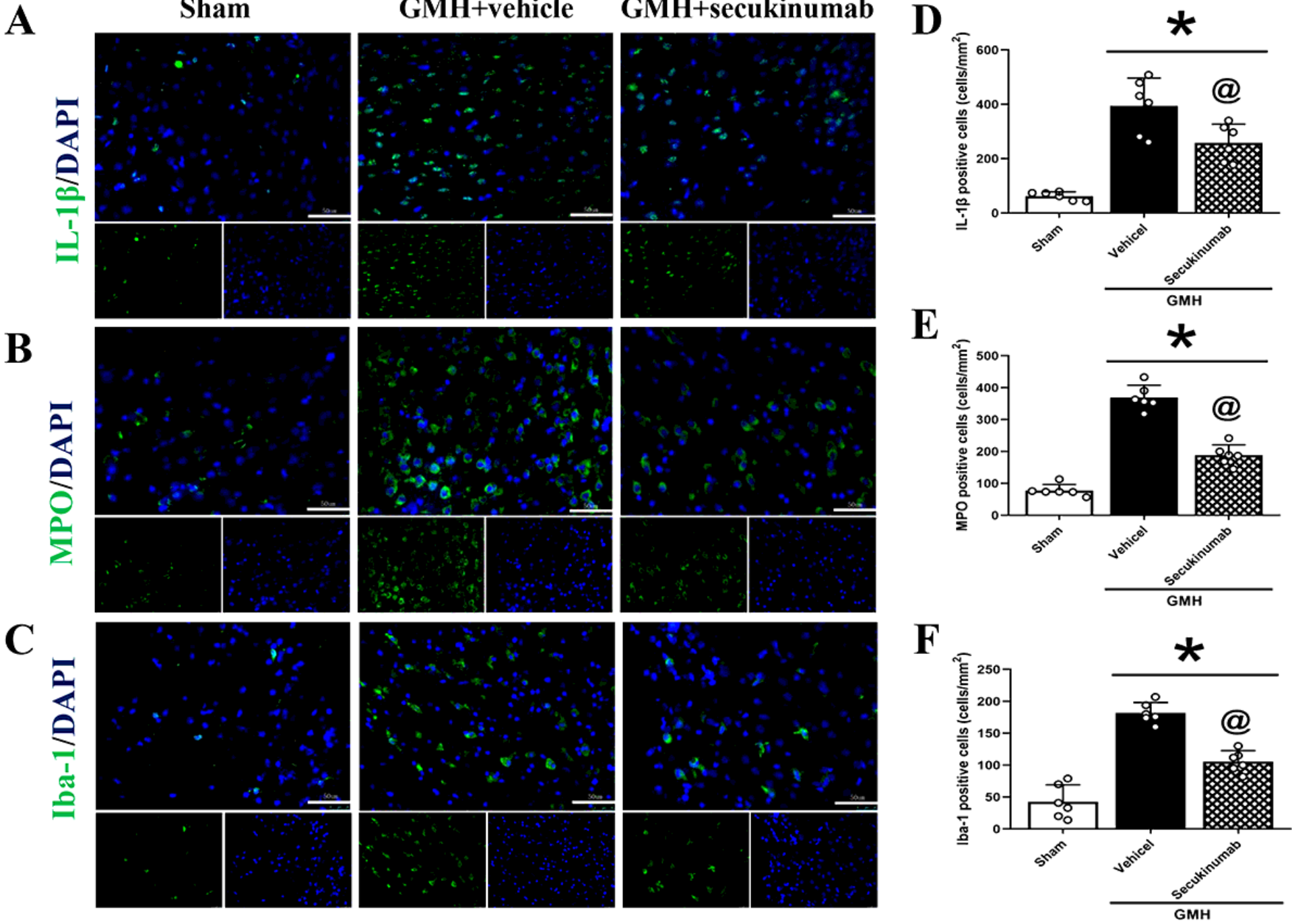

Figure 5

Secukinumab $(0.6 \mathrm{mg} / \mathrm{kg}, 1 \mathrm{~h}$, i.n.) treatment decreased the numbers of IL-1 $\beta+, \mathrm{MPO}+$, and $\mathrm{Iba}-1+$ cells in the perihematomal area at 24 hours after $\mathrm{GMH}$. (A, D) Colocalization of IL-1 $\beta$ and its number in each group; (B, E) Colocalization of MPO and its number in each group; $(C, F)$ Colocalization of Iba-1 and its number in each group. Scale bar $=50 \mu \mathrm{m},{ }^{*} \mathrm{P}<0.05$ vs. sham, mean $\pm \mathrm{SD}$, one-way ANOVA, Tukey's test $\mathrm{n}=$ 6/group. 
A

D

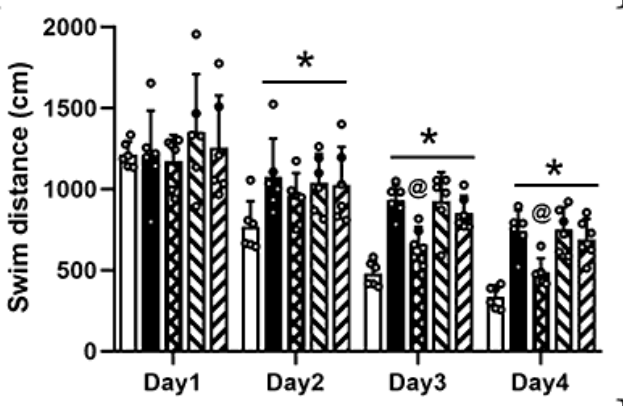

B

E

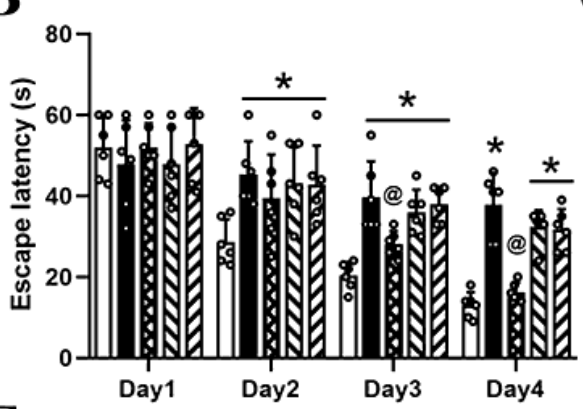

C

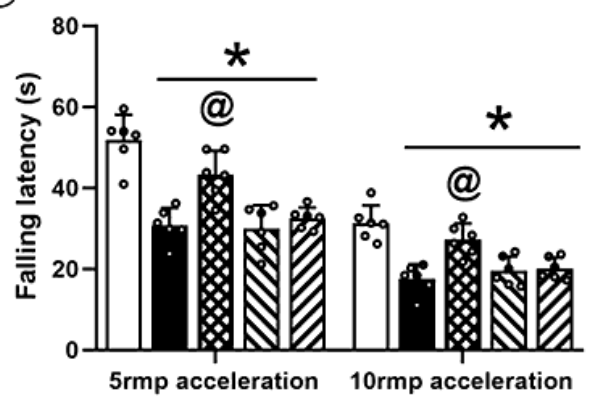

$\mathbf{F}$
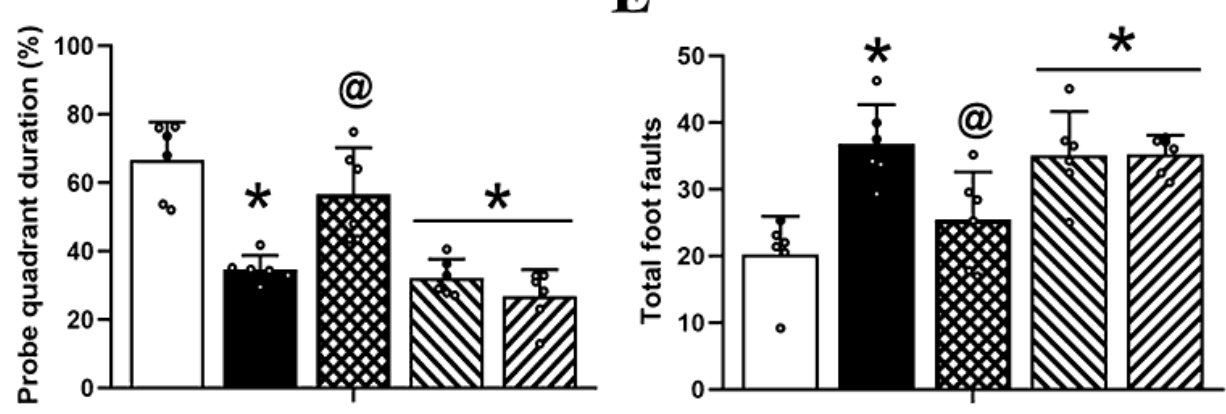

$\square$ Sham

- $\mathrm{GMH}+$ Vehicel

- $\mathrm{GMH}+$ Secuckinumab

凶 $\mathrm{GMH}+$ Secukukinumab+PMA

(2) $\mathrm{GMH}+$ Secukukinumab+ Ceramide $\mathrm{C} 6$

\section{G}

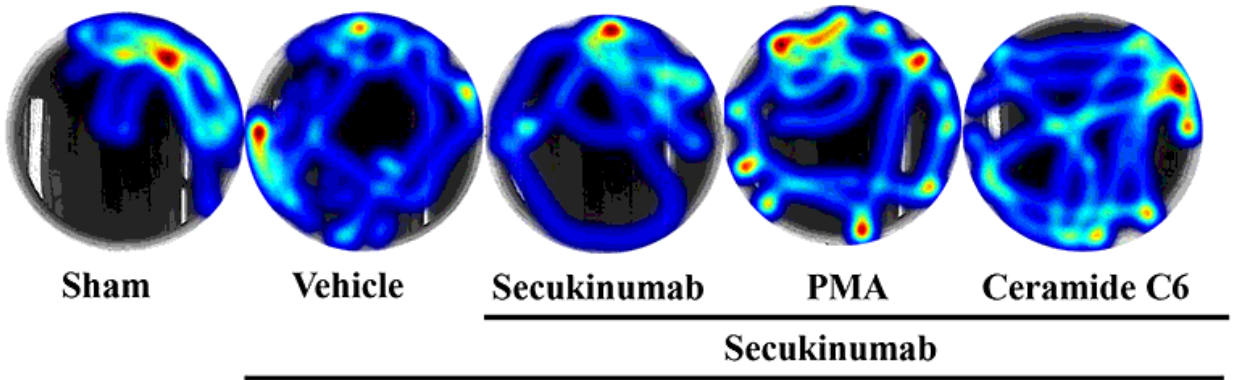

GMH

Figure 6

Secukinumab $(0.6 \mathrm{mg} / \mathrm{kg}, 1 \mathrm{~h}$, i.n.) treatment improved memory and motor function, PMA and Ceramide C6 offset its function at 22-27 days after GMH. (A, B, D, G) water Maze tests, (C) rotarod tests, (E) foot fault tests and $(F)$ graphic symbol. * $P<0.05$ vs. Sham, @P $<0.05$ vs. vehicle, mean $\pm S D$, two-way repeated measurement ANOVA for (A, B), one-way ANOVA for (C, D, E), Tukey's test, $n=6 /$ group. 
$\mathbf{A}$

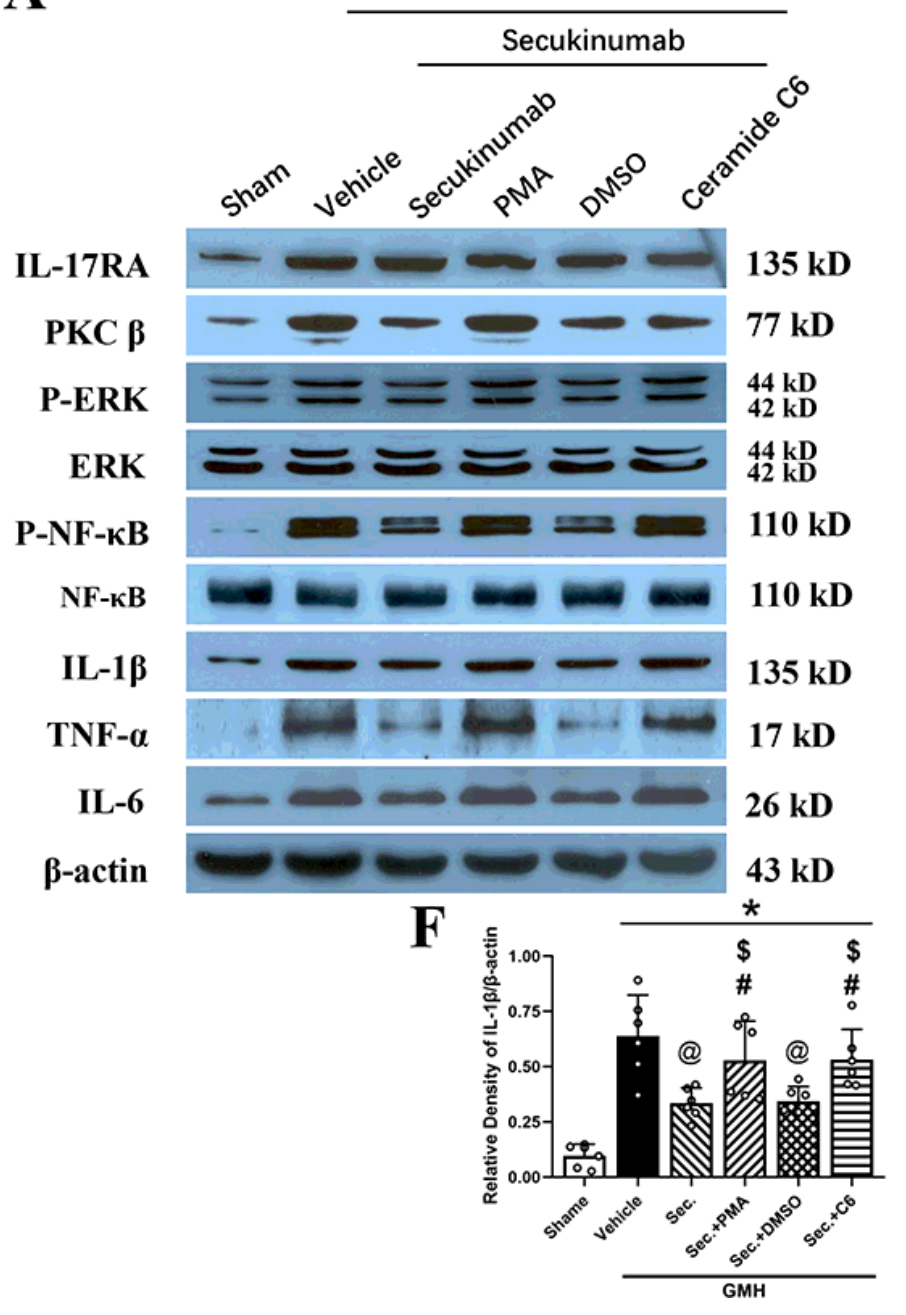

B

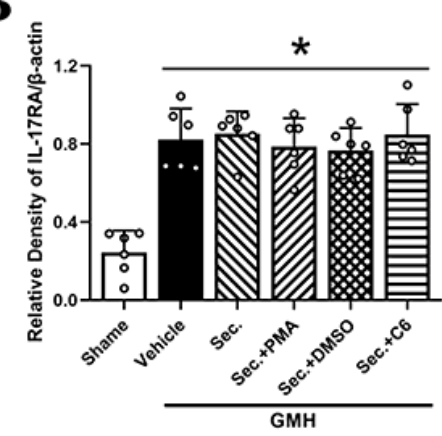

D

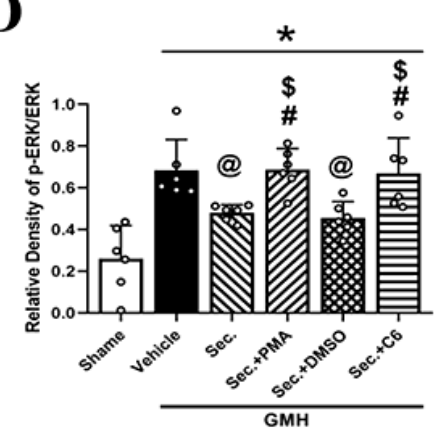

G

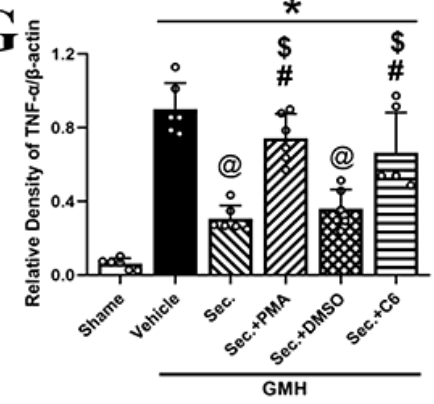

C

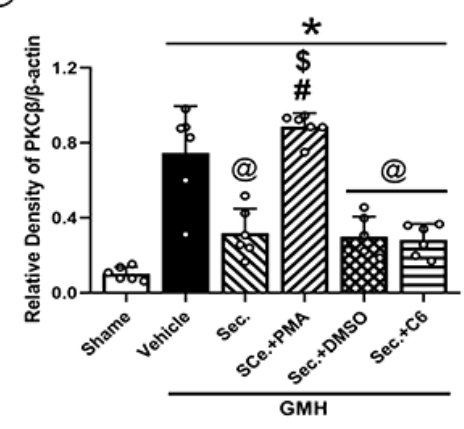

$\mathbf{E}$

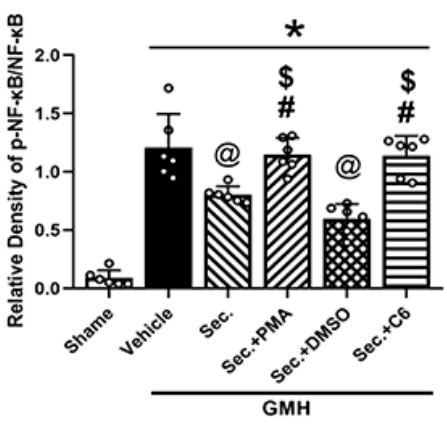

$\mathbf{H}$

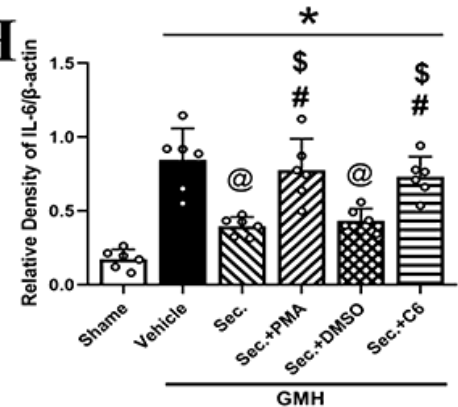

Figure 7

Secukinumab upregulated the proposed signaling pathway while PMA and Ceramide $\mathrm{C} 6$ reversed the effect of secukinumab after GMH. (A) The representative bands of IL-17RA, PKC $\beta, p-E R K, E R K, p-N F-k B$, NF-kB, IL-1 $\beta$, TNF- $\alpha$, IL- 6 and $\beta$-actin; (B-H) The quantitative analysis of IL-17RA, PKC $\beta, p-E R K, E R K, p-N F-$ kB, NF-kB, IL-1 $\beta$, TNF- $\alpha$, IL-6 intervened with secukinumab, PMA and Ceramide C6. ${ }^{*} P<0.05$ vs. Sham, $@ P<0.05$ vs. vehicle, $\# \mathrm{P}<0.05$ vs. secukinumab, $\$ \mathrm{P}<0.05$ vs. secukimumab + DMSO. Mean $\pm \mathrm{SD}$, one-way ANOVA, Tukey's test, $n=6 /$ group. 